\title{
COMPOSICIÓN Y ESTILO EN EL CANTO I DE LA HISTORIA DE LOS AMORES DE ABINDARRÁEZ Y JARIFA, ABENCERRAJES, DE FRANCESCO BALBI DA CORREGGIO (1593)
}

\author{
GABRIEL ANDRÉS \\ Università degli Studi di Urbino Carlo Bo \\ gabriel.andresrenales@uniurb.it
}

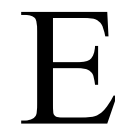

n los estudios sobre la épica española la Historia de los amores del valeroso moro Abindarráez y de la hermosa Jarifa, Abencerrajes... (Milán, 1593), de Francesco Balbi da Correggio, ha quedado siempre relegada a un papel marginal, siendo citada sin más en la mayoría de los estudios sobre la poesía épica hispánica o sobre las continuaciones del Abencerraje y la novela morisca, salvo un análisis más atento por parte de Giuseppe Mazzocchi, que no dejaba de considerar el poema como obra ajena al epos aurisecular: «sarà accettabile lo statuto di poema epico per la nostra operetta? Evidentemente no» $(1996: 553)^{1}$. Contribuye a juicios adversos como este, tal vez, el limitado perfil literario del autor, militar italiano dedicado a una reducida producción en lengua española de poesías panegíricas, además de una Verdadera relación... (1567) en prosa sobre los sucesos bélicos en la isla de Malta por esos años. También la moderada extensión del poema (689 octavas en 10 cantos) respecto a otros más extensos con indiscutido estatuto épico; así como su métrica y aparente estilo italianizzante con «tendenza al travestimento [...], piena di ibridismi» (Canonica, 2002: 68-69). Para una revisión de estas consideraciones, en este trabajo se atiende en particular modo a algunos aspectos de la obra relacionados con su composición y estilo, limitando en esta fase la atención al canto I, significativo por acoger elementos dispares, como veremos.

Desde el título se evoca, como línea temática, un espacio y un tiempo de convivencia y conflicto entre cristianos y musulmanes, algo que Mazzocchi (1996: 549) relacionaba con el trasfondo histórico de tensiones sociales de la época, que

1 Francisco Balbi de Correggio firma con este nombre españolizado sus obras, también esta Historia...

Edad de Oro, XXXIX (2020), pp. 177-189, ISSN: 0212-0429 - ISSNe: 2605-3314

DOI: https://doi.org/10.15366/edadoro2020.39.009 
culminarían más tarde en España con la expulsión de los moriscos (1609). Aunque, en realidad, Balbi no deja traslucir ninguna posición sobre esta cuestión; es más, su actitud en este caso se distancia de los grandes autores de la épica renacentista, abiertamente hostiles hacia lo musulmán, como sucede desde Ariosto, en la senda de una consolidada tradición humanista (Petrarca, Trionfo della Fama: II, 144; De vita solitaria: II, 460; etc.) que tachaba de canes a los ocupantes de Jerusalén, hasta llegar, entre otros, a Camões y Juan Rufo ${ }^{2}$. La única ocasión en que Balbi emplea el término «infiel» es para denunciar el conflicto en Flandes - «el Belga infiel» (Abin. VIII, 104: 3) - y la única vez que compara a algunos «como rabiosos canes» $(\mathrm{X}, 36: 3)$ lo hace denostando a los catalanes por un episodio en que se vio envuelto Juan Andrea D'Oria en una escala de su flota en Barcelona.

Como enseña Claudio Guillén (1988: 112), refiriéndose al Abencerraje primigenio en prosa, nos encontramos en realidad ante una práctica de poetización de la vida fronteriza andaluza, con elementos y personajes que resultan históricos, así Rodrigo de Narváez al servicio del príncipe Fernando en la toma de Antequera (1410) o la matanza del importante clan andalusí de los Abencerrajes en Granada (¿1472?), aunque no sea histórica precisamente la conexión de tales referencias entre sí dentro de la trama literaria del primer Abencerraje (Guillén, 1988: 114), ni tampoco en esta Historia de los amores... de Balbi, heredera de tales anacronismos ${ }^{3}$. El autor simplemente sigue la senda literaria de esta materia de ficción histórico-legendaria que tan amplia aceptación estaba teniendo incluso fuera de las fronteras ibéricas, como atestigua alguna traducción «manierista» italiana (García Dini, 1985), y que dentro de estas venía teniendo amplio eco. El mismo Rufo, limitándonos al ámbito de la epopeya, menciona al «Abencerraje el bravo y atrevido» (Aus. VII, 34: 5).

En cualquier caso, el canto I no entra aún de lleno en la materia de la ficción morisca preanunciada en el título, que solo va a desplegarse a partir del siguiente con las hazañas del alcaide Narváez y su primer enfrentamiento en batalla con Abindarráez. Este primero, que consta de 54 octavas, se abre con una estrofa de argumento o presentación de la trama, centrada en Martín I el Humano, rey de Aragón, que muere sin heredero y deja la gestión de su sucesión en manos de Fernando, infante de Castilla (véase Zurita, 2003: XI, cap. IX):

2 Ariosto: «[...] dove in carne abitò Dio onnipotente; / ch'ora i superbi e miseri cristiani, / con biasmi lor, lasciano in man de' cani» (Fur. XVII, 73: 6-8). Camões: «Não vedes a divina Sepultura / Possuída de Cães [...]» (Lus. VII, 9: 5-6). Juan Rufo, a propósito de los moriscos: «O pueblo desleal, linaje ingrato, / sacrílega nación, falsa, homicida [...]»(Aus. I, 76: 3-4).

3 Narváez, por ejemplo, presentado como alcaide de Álora, cuando solo en 1484 fue conquistado este enclave, décadas después de su muerte. 


\section{Argumento del canto I}

Don Martín de Aragón, rey verdadero, llegado ya al fin del curso humano y viéndose morir sin heredero, el mando a sus vasallos dio en mano para que hiciesen rey un caballero merecedor del cargo tan sovrano. Y así lo fue Fernando de Castilla, que cercaba Antequera, fuerte villa (I, 1).

Se despliega así una de las partes retóricas previstas en la praefatio o proemio de todo poema épico, la propositio (Curtius, 1984: II, 700), que preanuncia una de las líneas argumentales, la bélico-histórica en el campo cristiano, tratada precisamente en este canto I, mientras se deja para el II y siguientes la otra línea indicada en la sucesiva inuocatio, «el amor de un moro y de una mora» (I, 2: 8), «una amorosa hazaña» (I, 4: 8). De esta manera quedará configurada la trilogía de esas «armas, cortesías y amores» que Balbi anticipaba al dedicatario en los preliminares, evocando con ello los motivos del canon épico-caballeresco renacentista: «l'arme, gli amori, / le cortesie, l'audaci imprese» (Ariosto, Fur. I, 1: 1-2) 4 . A continuación, las siguientes estrofas organizan la parte central de la narratio en el canto I alrededor de una serie de núcleos temáticos estrechamente interrelacionados:

[10-18]: A partir de las últimas voluntades de Martín I de Aragón, se convoca a sus principales vasallos para que designen a un sucesor que lo merezca.

[19-26]: Se reúnen en cortes (Compromiso de Calpe), con la participación de san Vicente Ferrer en contra del aspirante Jaime, conde de Urgel; los nobles aceptan como rey a Fernando, por entonces a la conquista de Antequera para su sobrino, el joven rey castellano Juan II, de cuyo reino era regente temporal.

[27-32]: Fernando ante los nobles castellanos, cumpliendo el testamento del rey de Castilla, jura por rey a su sobrino, como regente hasta su mayoría de edad; todos los demás juran.

[33-44]: Fernando torna a la batalla de Antequera, donde luchaban caballerescamente moros y cristianos, y recibe allí a los embajadores de sus reinos (Aragón, Cataluña y Valencia), en visita de pleitesía a su nuevo monarca, que promete presentarse en sus reinos solo cuando tome Antequera; los enviados, admirados, deciden quedarse a ayudarle.

4 Dedicatoria de F. Balbi [a Muzio II Sforza Colonna]: «[...] vista su ilustrísima inclinación a las armas, cortesías y amores, siéndome venido a las manos la presente historia y viendo contener en ella todas las dichas partes, determiné volverla en verso [...]» (Abin., h. $\left.\mathrm{A}^{2} \mathrm{v}\right)$. 
[45-53:] Antequera se rinde y Fernando es clemente con los vencidos; antes de regresar a Zaragoza para su proclamación real — tras pasar por Castilla - , nombra alcaide de Antequera y de la vecina Álora al admirado caballero Rodrigo de Narváez.

La estrofa final $(I, 54)$ muestra, por su parte, recursos propios de la tópica del epílogo, con fórmulas de devoción al dedicatorio y de modestia (rusticitas), que emergen aquí como eco de las ya dirigidas en los preliminares a Muzio II Sforza:

$$
\begin{aligned}
& \text { Mas porque, mi Marqués, yo no pretendo } \\
& \text { de jamás daros un solo desgusto, } \\
& \text { de dar ya fin al primer canto entiendo, } \\
& \text { y si este, corto, no os diere buen gusto, } \\
& \text { no hay para qué los otros ir leyendo } \\
& \text { y al mal estilo el pago será justo. } \\
& \text { Mas reina en vos, señor, tal cortesía } \\
& \text { que vuestra musa suplirá a la mía (I, 54). }
\end{aligned}
$$

En otros cantos de este mismo poema la octava final de cierre-transición va a presentar elementos diversificados, empleando para ello otros recursos conclusivos característicos de la tradición de la epopeya, como recuerda Curtius (1984: II, 700) a propósito del esquema estructural de poemas latinos tardomedievales que podían incluir al final: un broche temático (epilogus) - como en Abin. V, 60 y VII, 59-, un diálogo del poeta con el libro (dialogica poete tetrastica), una recomendación de la obra (commendatio operis), o bien alguna consideración personal (confessio), en nuestro caso desde la senectud en que Balbi escribe reflexionando sobre sus avatares cuando servía a su protector en la corte española (Abin. IX, 50 y X, 75). En cualquier caso, es frecuente en estas octavas finales de transición una inconfundible matriz ariostesca (Goic, 1971):

Ma troppo è lungo ormai, Signor, il canto, e forse ch'anco l'ascoltar vi grava: sì ch'io differirò l'historia mia in altro tempo che più grata sia

(Ariosto, Fur. X, 115: 5-8).
[...] yo os contaré, Marqués, de quien la fama inchirá el mundo con más de un trofeo, en el siguiente canto, que es el cuarto, porque el tercero ya me tiene harto

(Balbi, Abin. III, 78: 5-8).

Con elementos recurrentes, como la manida referencia a la «pluma» cual sinécdoque de autoría en los versos: «[...] os lo contaré, Marqués, en suma./En tanto, cortaré mejor la pluma» (Abin. VII, 59: 7-8), al igual que en otra obra suya precedente, la Vida del ilustríssimo señor Octavio Gonzaga: «Mas porque está 
mi pluma muy cansada [...]» (Vida, 2014: 35 [24: 5]). Precisamente, este último poema del autor, en octavas acompañadas de glosa, es aquí determinante en la parte de inuocatio a Apolo y las musas (Abin. I: 2-9), según preveían el canon y la praxis del género:

\section{HISTORIA DE LOS AMORES... (I, 2)}

Ni del soberbio Ilión l'encendimiento, ni de la antigua Troya la caída, ni de la hermosa griega el robamiento, ni del troyano Eneas la partida, ni su contraste con la mar y viento, ni su llegada en el Lacio afligida quiere cantar mi Musa en esta hora, mas sí el amor de un moro y de una mora.
VIDA DE OCTAVIO GONZAGA... (2014:24[1])

No las troyanas armas coloradas en sangre griega por Héctor famoso, ni las del fiero Aquil ensangrentadas en la troyana, a quien fue tan dañoso; ni las astucias de Ulixes usadas, ni hecho por antiguo mentiroso: mas la vida, sucinta y brevemente, quiero contar de un varón excelente.

Parecido es, como puede apreciarse, el marcado hipérbaton con cláusulas paralelísticas encabezadas por lítote anafórica, así como el axis rítmico en sílabas $4 .{ }^{\mathrm{a}}-6 .{ }^{\mathrm{a}}$, aunque más marcado y efectivo estilísticamente en nuestra Historia (1593) que en la Vida (1581). El juego de correspondencias rítmicas con cláusulas bimembres de «epíteto» (axis en sílaba 4. ${ }^{\mathrm{a}}$ ) - «sustantivo» $\left(6 .^{\mathrm{a}}\right)$ en la primera parte de la octava se combina con remates de versos formados por sustantivos deverbales — «encendimiento» (usual por «incendio», véase $N D H E$ ), «caída», «robamiento» (arcaísmo, véase CORDE), «partida»- en un discreto juego de simetrías, aun a costa de tomarse toda la licencia poética que podía permitir nuestra tradición lírica en lo referido al empleo del apóstrofo, pues «l'encendimiento» alla maniera italiana (v. 1) es el único modo de declamar tal verso respetando el isosilabismo. Por lo demás, con esta octava Balbi sigue también la tradición tardorrenacentista del género, con un incipit formalmente parecido al de sus coetáneos —evidente en la Araucana de Ercilla: «No las damas, amor, no gentilezas / de caballeros canto enamorados, / ni las muestras, regalos y ternezas [...]» (Arau. I, 1:1-3)—, con esa estructura lógica del periodo («no... no... mas...»), construida a partir del juego de paralelismos en esquemas opositivos y empleo de anáforas o de quiasmos que, junto a otros recursos poéticos, venían también siendo típicos de los cancioneros corteses (Caravaggi, 1974: 15). Y si parecida es la inspiración que liga en esta octava la Historia (1593) a la Vida (1581), más estrecha resulta aún en las siguientes octavas de la invocación, que se configuran como autentico rifacimento del mismo episodio: 


$$
\text { HISTORIA ... (I, 3-5) }
$$

Mas por no haber a su placer gustada el agua de Helicona misteriosa que formó de un caballo la patada, salido de la sangre mostrüosa, no se atreve la pobre desdichada, sin socorro pidir, principiar cosa, y así, con reverencia y gran decoro, le pide a Febo y al Aonio coro:

$-\ll$ ¡Oh, rey de Delo!, si favor nunca diste a quien desease seguir tus pisadas y si por ruego jamás te moviste, tus orejas al mío no sean cerradas, y por el bien que a Dafne ya quisiste dispone a las hermanas celebradas y juntos todos dadme hoy tal maña que contar pueda esta amorosa hazaña.

En la cual se verá no solamente en tierna edad un amor muy subido y con cuánto valor muy cuerdamente resistieron al fuego de Cupido, hasta que descubrieron ciertamente, cada cual mortalmente estando herido, que podían a la fin hallar remedio, pues Amor les mostró con tiempo el medio.

$$
V_{I D A} \ldots(2014: 25-26 \text { [2-4]) }
$$

Mas siendo nuevo y no habiendo gustado el agua de Helicona misteriosa (que salió do el caballo hobo pisado, salido de la sangre mostrüosa), de mí mismo jamás sería osado de dar principio a cosa tan famosa, si no pidiese, como pido y debo, a las Nueve socorro, y al gran Febo:

Apolo, pues, si favor jamás diste a quien deseasse de cantar grande hecho, y si por ruego jamás te moviste, muévate el que sale de mi pecho. Y por el bien que a Dafne ya quisiste, por él te ruego, y no sea sin provecho, que tú con tus hermanas me deis maña para contar esta famosa hazaña.

En la cual se verá no solamente en tierna edad un valor muy crecido y en joventud después tan diferente, como a su ilustre estado era debido; llegado a ser hombre ciertamente $[\ldots]$

Se despliega en estas estrofas el conocido topos del escenario mitológico en el que se sitúa la fuente de inspiración poética (Curtius, 1984: 324-348), según declara el mismo Balbi en su glosa a la octava 2 de la Vida de Octauio Gonzaga ${ }^{5}$. Los elementos de tradición ariostesca son constantes en estos versos de la invocación, a partir de las referencias a Helicona, montaña consagrada a Apolo y a las Musas en la región de Aonio, en Beocia, cerca del monte Parnaso, donde mana la fuente Hipocrene tras haber apoyado Pegaso su pezuña en ella ${ }^{6}$. Del mismo modo,

«Siendo embiado Perseo, hijo de Júpiter y Dánae por Acrecio contra las Gorgonas, cortada la cabeça a Medusa, con el favor de Mercurio y Palas, sus hermanos, salió de la sangre d'ella un caballo alado, el cual en pisando la tierra salió una fuente de la pisada, la cual fuente tomaron para su morada las nueve Musas hermanas de Febo, hijo de Júpiter y de latona. Ovidio, Metamorfosis» (Vida, 2014:25).

6 Elementos temáticos coincidentes con esta tradición ariostesca — «[...] le pide a Febo y al Aonio coro» (Abin. I, 3: 8); «[...] grata a Febo e al santo Aonio coro» (Ariosto, Fur. XLVI, 3: 8) - se encuentran igualmente en Camões o en Rufo: «[...] qual fe' il cavallo alato uscir del monte,/non so se di Parnasso o d'Elicano» (Fur. XLII, 91: 3-4) y «Por que de vossas águas Febo ordene / 
en las estrofas del canto I posteriores prosigue esta estrecha ligazón con el modelo ariostesco, muy evidente, por ejemplo, en el cruce de referencias genealógicas entre Balbi (Abin. I, 7: 1-5: «De la alta sangre que trae el colubro / [...] dende el Tamis y Ródano al mar Rubro, / del gran nombre romano alta Colona, / dominó la primera el reino Insubro») y Ariosto (Fur. XII, 63: 1-5: «E Moro e Sforza e Viscontei colubri / lei viva, formidabili saranno / da l'iperboree nievi ai lidi rubri, / [...] lei morta, andran col regno degl'Insubri»).

Y junto a esta comunidad de motivos, el poema de Balbi comparte otros rasgos estilísticos con los autores épicos ibéricos que van más allá del calco temático, en esa especie de koiné estilística, propia no solo de los textos épico-caballerescos, y que en el caso de Francesco Balbi es fruto de una cultura literaria, si no elevada, próxima al menos a la de otros literatos del periodo. Véase, en este sentido, otra breve muestra de soluciones empleadas por el autor a lo largo de este canto I que coinciden con la praxis literaria de sus modelos o de sus coetáneos:

DEGEN[E]RA (Abin. I, 8:2): «[...] que no degenra de insubro y romano»; «degenera», latinismo, «quando uno desdize de la nobleza de sus antepassados»(Cov.), con síncopa por metaplasmo vocálico. El término se halla también en el primer Abencerraje, aunque solo en la versión del Inventario: «por parecer a aquellos donde vengo y no degenerar de la alta sangre de los Abencerrajes» (Carta del Abencerraje Abindarráez al alcaide de Álora, El Abencerraje, 2017: 57).

EL QUE RIGE EL CIELO (Abin. I, 9:1-5): «Y así espero en el que rige el cielo [...]». Estilema tradicional ya en Ariosto, «abbia chi regge il ciel cura del resto» (Fur. XXII, 57: 3); también en Camões, «Senão no sumo Deus que o Céu regia» (Lus. III, 43: 2); como en Rufo: «a quien el sumo Rey que rige el cielo» (Aus. I, 5: 2).

MEMORANDO (Abin. I, 34: 5-6): «había contino trance memorando / entre cristiano y moro señalado». En Ariosto, «con alto e memorando / segno» (Fur. XX, 79: 5-6), como en el mismo Balbi, Vida..., «No se acabó el trance memorando: / que de caballos capitán fue digno» (Vida, 2014: 25 [30: 5-6]); también en la cervantina Comedia famosa del gallardo español, «iOh Robledo, / verdadero y memorando, / y cuánta verdad dijiste!» (2001:f. 16v; vv. 727-729).

Francesco Balbi conoce, pues, el canon tradicional de la poesía épica anterior a Torquato Tasso y posee también una suficiente competencia lingüística y literaria en español para lanzarse a la composición de un texto poético de estas

Que não tenham enveja às de Hipocrene» (Camões, Lus. I, 4: 7-8). También en el empleo del topos horaciano del attentam aurem — «Nisi dextro tempore Flacci / Verba per attentam non ibunt Cæsaris aurem» (Sátiras, II: 1) —, en Ariosto — «[...] vi farò udir, se voi mi date orecchio» (Fur. I, 4: 6) - , como en Balbi — «[...] tus orejas al mío no sean cerradas» (Abin. I, 4: 4) - o también en Ercilla: «dad orejas, Señor, a lo que digo» (Arau. I, 5: 7). 
características. La Historia de los amores de Abindarráez y Jarifa, Abencerrajes aparece desde el canto I como proyecto literario bajo el signo del epos renacentista y como tal cabe considerarlo, dentro de ese margen de flexibilidad temática y estructural que caracteriza a la épica hispánica del periodo (Pierce, 1968: 264), calibrando cuanto sea necesario su valía, ciertamente, respecto al resto de la producción poética de este signo, pues, en efecto, no son pocas las singularidades del texto de Balbi que distorsionan el modelo.

Algunas de estas son de tipo compositivo, como se aprecia en esa técnica de rifacimento de la que se sirve el autor en el proemio del canto I, reutilizando materiales concebidos previamente a mayor gloria de otro noble italiano, Ottavio Gonzaga, también al servicio de la monarquía hispánica (Vida ..., 1581) 7 . Un procedimiento que tal vez pudiera aplicarse igualmente al canto VIII, cuya desproporcionada extensión (120 octavas), a propósito de Felipe II y las glorias de la monarquía hispánica, permite suponer que contenga en alguna medida las 88 octavas de una pieza precedente de temática similar del mismo Balbi hoy desconocida: Breve discurso al grandissimo y muy poderoso Phelipo Segundo, Rey de las Espannas, $y$ Monarca del Occidente (1585).

Otras son singularidades de tipo métrico y lingüístico-estilístico que no se encuentran en otros poetas épicos del periodo. Algunas pudieran parecer meros italianismos, que se confunden con arcaísmos del español o con licencias poéticas aceptables para los lectores ibéricos de la época. Así construcciones como «por no haber a su placer gustada / el agua [...]» (Abin. I, 3: 1-2), concordante entre participio y complemento, no eran insólitas en español hasta principios del siglo XVI (Rodríguez Molina, 2016) ${ }^{9}$. Del mismo modo, ocasionales construcciones de «artículo + posesivo + sustantivo» — «porque muerto había un su hermano» (I, 20: 7); «un suyo embajador» (I, 44: 5) — son un posible recurso estilístico autóctono por trasposición desde fuentes, como crónicas y romances, que ya contenían ese lenguaje arcaizante (Lapesa, 2000: I, 413), o quizá por voluntad de «afectación» literaria, según señalaba Juan de Valdés en su Diálogo de la lengua a propósito de los libros de caballerías (Terracini, 1964; Profeti, 1966: 51). Igualmente, el apóstrofo de artículo determinado ante sintagma nominal — «l'alto pimpollo» (I, 6: 6); «l'alma rindió» (I, 18: 4); «l'Infante gobernase» (I, 32: 2); «l'amor mostrando»

7 Baldissera (2014: 17-18) identifica a su vez otro rifacimento previo de algunos pasajes de esta Vida (1581) respecto a un precedente texto de Pedro Cornejo del que se sirve Balbi: Origen de la ciuil disension de Flandes... (Torino, 1580).

8 La distribución estrófica en los diez cantos de la Historia de los amores... es la siguiente: I (54 oct.), II (58), III (78), IV (69), V (60), VI (66), VII (59), VIII (120), IX (59), X (75). Palau (1948-1977: II, n. $\left.{ }^{\circ} 22.300\right)$ y Simón Díaz (1950-1993: VI, n. $\left.{ }^{\circ} 2.234\right)$ ofrecen noticias de este perdido texto poético publicado al parecer en Milán por el mismo impresor, Pacifico Pontio (1585).

9 Bien pudiera tratarse de variante por culpa del tipógrafo italiano, pues resulta con solución no concordante en la Vida ... (2014: 25 [2:1-8]): «no habiendo gustado / el agua». 
(I, 53: 6) — permite siempre en el poema de Balbi la isometría del verso, sin resultar extraño a la tradición métrica y literaria ibérica.

Por lo que se refiere a la métrica, Balbi sigue básicamente la octava ariostesca sin grandes torsiones estructurales respecto al modelo (limitado empleo del encabalgamiento, amplio recurso a sinalefa y dialefa, estilemas formulísticos que facilitan isosilabismo y rima, relevancia del dístico final de octava como remate estrófico, etc.). Sin embargo, incluye experimentos métricos que son más propios de cierta poesía cortés o de certamen, como el empleo de la autorrima triple (véase en la ya citada octava I, 5, con rimas: «solamente-cuerdamente-ciertamente...»y, en rima interna, «mortalmente»); así como el uso de autorrimas trabadas (alternas, cruzadas o entrelazadas) en versos pares-impares: «Y más les dijo con voz alta y "clara" / que les agradecía el buen "deseo". / Y porque conociesen a la "clara" / en él contino un mismo "deseo", / juraba por su fee, muy limpia y "clara", / que siempre tendría aquel mismo "deseo"» (Abin. I, 31: 1-6). Esta última modalidad será un recurso métrico-estilístico repetido en el poema, especialmente en las octavas conclusivas de algunos cantos (II, 48; III, 77; VI, 62 y 63; VIII, 50); como sucedía ya en una de las estrofas finales de su anterior Vida de Octavio Gonzaga (1581):

Y porque al fin es este cuento raro, en quien se ve valor, amor y esfuerzo, a vos lo he dedicado, ;oh, Muzio raro en valor natural, amor y esfuerzo! Y si no fuérades hoy día tan raro, de mí mismo jamás tuviera esfuerzo [...]

(Abin. X, 10: 1-6).
Y porque esta historia ha sido rara en quien se encierra amor, valor y esfuerço, a vos la he dedicada, al mundo rara en amor en saber, valor y esfuerço, y si no fuérades cierto tan rara de mí mismo jamás tuviera esfuerço $[\ldots]$

(Vida, 2014: 91 [170: 1-6]).

A diferencia del resto de poetas épicos renacentistas y coetáneos, el autor emplea aquí ampliamente este tipo de juegos métricos (autorrimas dobles y triples, rimas trabadas...), hasta el punto de servirse de ellos aproximadamente en el $13 \%$ de las estrofas del poema, y en algunos de los cantos en manera particular:

$$
\text { cantos: I - II - III - IV - V - VI - VII - VIII - IX - X }
$$

total de estrofas con autorrimas: $4-6-6-11-3-19-4-19-17-3$

Francesco Balbi aparece, en definitiva, como autor que ha completado su bagaje medio de cultura literaria italiana con lecturas hispánicas más bien tradicionales en lo poético, como confirma, a su vez, la también ocasional inclusión en el canto I de su Historia de los amores... de juegos de políptoton — «usaré $[\ldots]$ la fuerza $[. .$.$] para pintar [. .$.$] la fuerza [. .$.$] que en mi virtud [. .$.$] esfuerza»$ (I, 9: 6-8); «reinaba de reinar» $(\mathrm{I}, 42: 2) \ldots-$, en la línea del «redoblado» y otras recurrencias parecidas, propias de la poesía cortés, que señalara Juan del Encina 
en su Arte de poesía castellana (1978: I, 28), sin descartar también su posible uso como arcaísmo de gusto caballeresco (Profeti, 1966: 67-68). Y si a ello unimos que la única autoridad literaria citada explícitamente en el canto I es nuestro tan hispánico Juan de Mena - «Don Juan fue este el segundo sonado, / que Mena mereció le celebrase / cuando dijo que Júpiter del suelo / tanto le dio como tomó él del cielo» (I, 32: 5-8 ${ }^{10}$-, tendremos un cuadro del horizonte literario en el que se movía Francesco Balbi, limitado pero complejo y sugestivo a un tiempo, con un hibridismo que es lingüístico en más de una ocasión, pero sobre todo literario y cultural entre las dos orillas del Mediterráneo.

\section{BiBLIOGRAFÍA}

ABIN. $=$ véase BALBI DA CoRregGio, Francesco (1593). Historia de los amores...

ARAU. $=$ véase Ercilla, Alonso de (1993). Araucana.

Ariosto, Ludovico (1964). Orlando Furioso. Marcello Turchi (ed.), Edoardo Sanguineti (intr.). Milano: Garzanti.

Aus. = véase Rufo, Juan (2011). La Austríada.

BAlBi da Correggio, Francesco (1567). La verdadera relacion de todo lo que este año de m.d.lxv ha sucedido en la Isla de Malta. Alcalá de Henares: Iuan de Villanueua (reed. 1568, Barcelona).

Balbi da Correggio, Francesco (1593). Historia de los amores del valeroso moro abinde Araez y de la hermosa xarifa Aben çerases. Y la battalla que hubo con la gente de Rodrigo de Narbaez ala saçon, Alcayde de Antequera, y Alora, y con el mismo Rodrigo. Vueltos en verso... Milan: Pacifico Ponçio [Da Ponte].

BAlBi da Correggio, Francesco (2014). La vida del illustríssimo señor Octavio Gonzaga [1581]. Andrea Baldissera (ed.). En Paolo Pintacuda (ed.), Le vie dell'epica ispanica. Lecce / Rovato: Pensa Multimedia, pp. 11-93.

Camões, Luís de (2000). Os Lusíadas. Álvaro Júlio da Costa Pimpão (ed.), Aníbal Pinto de Castro (intr.). Lisboa: Instituto Camões, 4 . $^{\mathrm{a}}$ ed. $<$ http://cvc.instituto-camoes.pt/ conhecer/biblioteca-digital-camoes/explorar-por-autor.html?aut=182> [Consulta: 06/07/2019].

CANonica, Elvezio (2002). «Venere translingue: scrittura amorosa in spagnolo di autori italiani, tra Cinque e Seicento». En Domenico Antonio Cusato y Loretta Frattale (coords.), Testi specialistici e nuovi saperi nelle lingue iberiche. Atti del XX Convegno AISPI. Messina: Andrea Lippolis, I, pp. 59-70 <https://cvc.cervantes.es/ literatura/aispi/testi.htm> [Consulta: 03/05/2019].

Caravaggi, Giovanni (1974). Studi sull'epica ispanica del Rinascimento. Pisa: Università di Pisa.

\footnotetext{
10 Juan de Mena, al servicio de Juan II como secretario de cartas latinas, con su Laberinto de Fortuna será modelo para la consolidación posterior de una lengua culta literaria española (Lida de Malkiel, 1952) y aun portuguesa con Camões (Extremera, 2013). Aquí la referencia corresponde al incipit de la obra: «Al muy prepotente don Juan el segundo, / aquél con quien Júpiter tuvo tal zelo / que tanta de parte le fizo del mundo / quanta a sí mesmo se fizo del çielo» (1995: 93).
} 
Cervantes Saavedra, Miguel de (2001). Comedia famosa del gallardo español. Florencio Sevilla Arroyo (ed.). Alicante: Biblioteca Virtual Cervantes $<$ http://www.cervantesvirtual.com/nd/ark:/59851/bmc9p2z9> [Consulta: 16/09/2019].

CORDE = Real Academia Española, Corpus diacrónico del español <http://www.rae.es/ recursos/banco-de-datos/corde> [Consulta: 09/06/2019].

Cov. = Covarrubias Horozco, Sebastián de (2006). Tesoro de la lengua castellana o española. Ignacio Arellano y Rafael Zafra (eds.). Madrid / Frankfurt am Main: Iberoamericana / Vervuert.

CurTius, Ernst Robert (1984). Literatura europea y Edad Media Latina. Ciudad de México / Madrid / Buenos Aires: Fondo de Cultura Económica.

Di stefano, Giuseppe (ed.) (1966). El libro del famoso e muy esforçado cavallero Palmerín de Olivia. Pisa: Università di Pisa.

$D R A E=$ Real Academia Española, Diccionario de la lengua $<$ http://dle.rae.es/> [Consulta: 09/06/2019].

El Abencerraje y La Hermosa Jarifa (2017). Eugenia Fosalba (ed.). Barcelona / Madrid: Círculo de Lectores / Espasa Calpe.

Encina, Juan del (1978). Arte de poesía castellana. En Ana M. ${ }^{a}$ Rambaldo (ed.). Obras completas. Madrid: Espasa-Calpe.

Ercilla, Alonso de (1993). Araucana. Isaías Lerner (ed.). Madrid: Cátedra.

Extremera TAPia, Nicolás (2013). «Contribuciones al léxico culto de Juan de Mena y Luis de Camoens». Revista de Filología Románica, 20: 1, pp. 75-94.

Fur $=$ véase Ariosto, Ludovico (1964). Orlando Furioso.

GARCÍA DINI, Encarnación (1985). Una traducción italiana manierista de «El abencerraje». Napoli: Istituto Universitario Orientale.

Gorc, Cedomil (1971). «La tópica de la conclusión en Ercilla». Revista Chilena de Literatura, 4 , pp. 17-34.

Gotor, José Luis (1993). «Ezio Levi, un hispanista erudito». En L'apporto italiano alla tradizione degli studi ispanici, Atti del Congresso AISPI (Napoli, 30 genn.-1 febb. 1992). Roma: Instituto Cervantes, pp. 71-84.

GuILlÉn, Claudio (1988). «Individuo y ejemplaridad en el Abencerraje». En El primer siglo de oro. Estudios sobre géneros y modelos. Barcelona: Crítica, pp. 109-153.

LAPESA, Rafael (2000). Estudios de morfosintaxis histórica del español. Rafael Cano Aguilar y M. ${ }^{\text {a }}$ Teresa Echenique Elizondo (eds.). Madrid: Gredos.

Lida de Malkiel, María Rosa (1952). La idea de la fama en la Edad Media castellana. Ciudad de México / Madrid / Buenos Aires: Fondo de Cultura Económica.

Lus. = véase CAMÕES, Luís de (2000). Os Lusíadas.

Mazzocchi, Giuseppe (1996). «Sulla Historia de los amores del valeroso moro Abindarráez di Francesco Balbi da Correggio». En Simone Albonico et alii (eds.), Per Cesare Bozzetti. Studi di letteratura e filologia italiana. Milano: Mondadori, pp. 547-572.

MenA, Juan de (1995). Laberinto de Fortuna. Maxim P.A.M. Kerkhof (ed.). Madrid: Castalia.

NDHE $=$ Real Academia Española, Nuevo diccionario histórico del español $<$ http://www.rae es/recursos/diccionarios/nuevo-diccionario-historico > [Consulta: 09/06/2019]. 
Palau y Dulcet, Antonio (1948-1977). Manual del librero hispano-americano. Madrid: Librería Palau.

PIERCE, Frank (1968). La poesía épica del Siglo de Oro. Madrid: Gredos.

Profetr, Maria Grazia (1966). «Afectación e Descuido nella lingua del Palmerín». En Studi sul Palmerín de Olivia, III. Saggi e ricerche. Pisa: Università di Pisa, pp. 45-73.

Rodríguez MolinA, Javier (2016). «Patrones de variación en la concordancia del participio en español antiguo». En En torno a 'haber'. Construcciones, usos y variación desde el latín hasta la actualidad. Bern: Peter Lang, pp. 417-467.

Rufo, Juan (2011). La Austríada. Ester Cicchetti (ed). Como: Ibis.

Simón DíAz, José (1950-1993). Bibliografia de la Literatura Hispánica. Madrid: Instituto «Miguel de Cervantes» de Filología Hispánica.

TERRACINI, Lore (1964). Tradizione illustre e lingua letteraria nella Spagna del Rinascimento. Roma: Tip. PUG.

Vida $=$ véase BAlBI DA Correggio, Francesco (2014). La vida de ... Octavio Gonzaga .

ZuritA, Jerónimo (2003). Anales de la Corona de Aragón. Á. Canellas López (ed.). Zaragoza: Institución Fernando el Católico <https://ifc.dpz.es/publicaciones/ebooks/ id/2448> [Consulta: 21/06/2019].

Recibido: 29/09/2019

Aceptado: 12/02/2020 


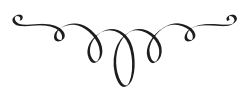

Composición y eStilo en el CANTO I DE la HiSTORIA DE LOS AMORES DE ABINDARRÁEZ y Jarifa, AbenCerRajes, de Francesco Balbi da Correggio (1593)

RESUMEN: La continuación del Abencerraje en verso publicada en Milán (1593) por el italiano Francesco Balbi da Correggio ha quedado relegada a un papel marginal en los estudios sobre la poesía épica hispánica o sobre las versiones del conocido relato morisco y merece una mayor atención que nos permita revisar, parcialmente al menos, algunos juicios críticos que se han generalizado sobre esta obra. En este trabajo se atiende a algunos aspectos relacionados con su composición y estilo, en particular en el significativo canto I. Se observa cómo, junto a un consolidado bagaje literario de épica italiana renacentista (Ariosto), el autor ha asimilado en modo sui generis lecturas hispánicas tradicionales ajenas a la praxis de los autores épicos coetáneos.

Palabras Clave: Poesía épica española, poesía del Siglo de Oro, abencerraje, literatura morisca.

\section{Composition and Style in the Ahant I of the Historia DE lOS AMORES DE} AbindarRÁEZ y Jarifa, AbenCERRaJes, By Francesco Balbi da Correggio (1593)

ABSTRACT: The continuation of the Abencerraje published in Milan (1593) by Francesco Balbi da Correggio has been relegated to a marginal role in the studies related to Hispanic epic poetry or in the versions of the well-known Moorish novella; however, it probably deserves greater attention in order to review, partially at least, some critical judgments that have become widespread about this literary work. This article addresses some aspects related to its composition and style, in particular in the Chant I, which is significant for hosting relevant elements. It is observed how, together with a consolidated literary baggage of the Italian epic genre during the Renaissance (Ariosto), the author has also assimilated traditional Hispanic readings outside the praxis of contemporary epic authors.

Key words: Spanish Epic Poetry, Golden Age Poetry, Abencerraje, Moorish Literature. 